\title{
New drug classes for the treatment of partial onset epilepsy: focus on perampanel
}

This article was published in the following Dove Press journal:

Therapeutics and Clinical Risk Management

6 July 2013

Number of times this article has been viewed

Jerry J Shih'

William O Tatum'

Leslie A Rudzinski²

'Department of Neurology, Mayo Clinic, Jacksonville, FL, USA;

2Department of Neurology, Emory

University, Atlanta, GA, USA
Correspondence: Jerry J Shih

Department of Neurology, Mayo Clinic, Jacksonville, FL 32224, USA

Tel +l 9049532498

Email shih.jerry@mayo.edu
Abstract: Perampanel (2-[2-oxo-1-phenyl-5-pyridin-2-yl-1,2-dihydropyridin-3-yl] benzonitrile hydrate) is the latest in the line of new antiepileptic drugs with a novel mechanism of action. Perampanel inhibits $\alpha$-amino-3-hydroxy-5-methyl-4-isoxazole-propionic acid (AMPA)-induced increases in intracellular $\mathrm{Ca}^{2+}$ and selectively blocks AMPA receptor-mediated synaptic transmission, thus reducing neuronal excitation. Three Phase III multicenter, randomized, doubleblind, placebo-controlled trials demonstrated the efficacy and good tolerability of perampanel as adjunctive treatment in patients with refractory partial-onset seizures. The drug is approved for use in the European Union and United States, with expected release onto the American market in June-September 2013, pending US Drug Enforcement Agency classification. The pharmacology of perampanel offers potential as more than just another new antiepileptic drug. This first-in-class drug will provide another option for practitioners of rational polytherapy. As an AMPA-receptor antagonist, perampanel may possess antiepileptogenic properties in addition to its demonstrated antiseizure properties.

Keywords: perampanel, mechanism of action, efficacy, review

\section{Introduction}

The goal of antiepileptic drug (AED) treatment is the elimination of seizures without the presence of treatment-emergent side effects that lower quality of life. Over the past 30 years, several new AEDs have been developed and ultimately marketed in the United States, European Union, and Asia with that objective in mind. To a large degree, the newer drugs such as gabapentin, lamotrigine, and levetiracetam are better tolerated than older agents such as phenytoin and phenobarbital. ${ }^{1}$ However, efficacy of the newer drugs, as defined by seizure control, was not demonstrably different from drugs available before $1980 .^{2}$ The proportion of patients diagnosed with epilepsy that subsequently become medically refractory remains at around $30 \%$, despite the introduction of over 15 new antiepileptic drugs in the last 20 years. ${ }^{3}$ Many clinicians began using combinations of drugs with different mechanisms of action with the belief or hope that "rational polytherapy" may produce better seizure control. ${ }^{4}$ Recognizing that many of the older drugs such as phenytoin and carbamazepine were either sodium channel modulators or gamma-aminobutyric acid (GABA) agonists (benzodiazepines, phenobarbital), preclinical drug testing programs in the 1980s and 1990s focused on developing compounds with novel mechanisms of action.

Several new AEDs with novel mechanisms of action have come to market in the United States and the European Union (EU) in the past 3 years. Retigabine/ezogabine (Potiga ${ }^{\circledR}$, GlaxoSmithKline, London, UK) is a new AED that has a potent and broad spectrum of activity 
in various anticonvulsant animal models. Retigabine/ezogabine has a unique mechanism of action, consisting mainly of activation of the neuronal M-current by targeting $\mathrm{K}_{\mathrm{V}} 7.2$ channels. Enhancing the M-current elevates the seizure threshold and protects against seizures, mainly by preventing rapid recurrent membrane depolarization. ${ }^{5}$ Another AED with a novel mechanism of action is lacosamide (Vimpat ${ }^{\circledR}, \mathrm{UCB}$ Brussels, Belgium), which has applications for epilepsy and neuropathic pain. Lacosamide differs from other AEDs that act on voltagegated sodium channels in that it selectively enhances the slow inactivation component at these channels and also interacts with collapsin-response mediator protein $2 .^{6}$

\section{Development history}

Perampanel (Fycompa ${ }^{\circledR}$; Eisai Inc, Woodcliff Lake, NJ) is the latest in the line of new AEDs with novel mechanisms of action (Figure 1). Perampanel is a result of a focused discovery program at Eisai research laboratories in London and Ibaraki Prefecture, Japan. ${ }^{7}$ Chemical structures were explored using high throughput screening assessing inhibition of $\alpha$-amino-3-hydroxy-5-methyl-4-isoxazole-propionic acid (AMPA)-induced cortical neuron death and AMPA-induced calcium influx with a membrane permeant calcium-sensitive dye. Structure-activity relationships of compounds based on various core structures were investigated, with the pyridone core eventually being selected as the preferred scaffold. Among a number of potential clinical candidate compounds built on this core, perampanel was selected for further development. ${ }^{8}$ The drug was initially tested as an antiparkinsonian compound. Because of its pharmacologic properties, perampanel was a logical candidate to assess for an anticonvulsant effect. It has subsequently undergone extensive

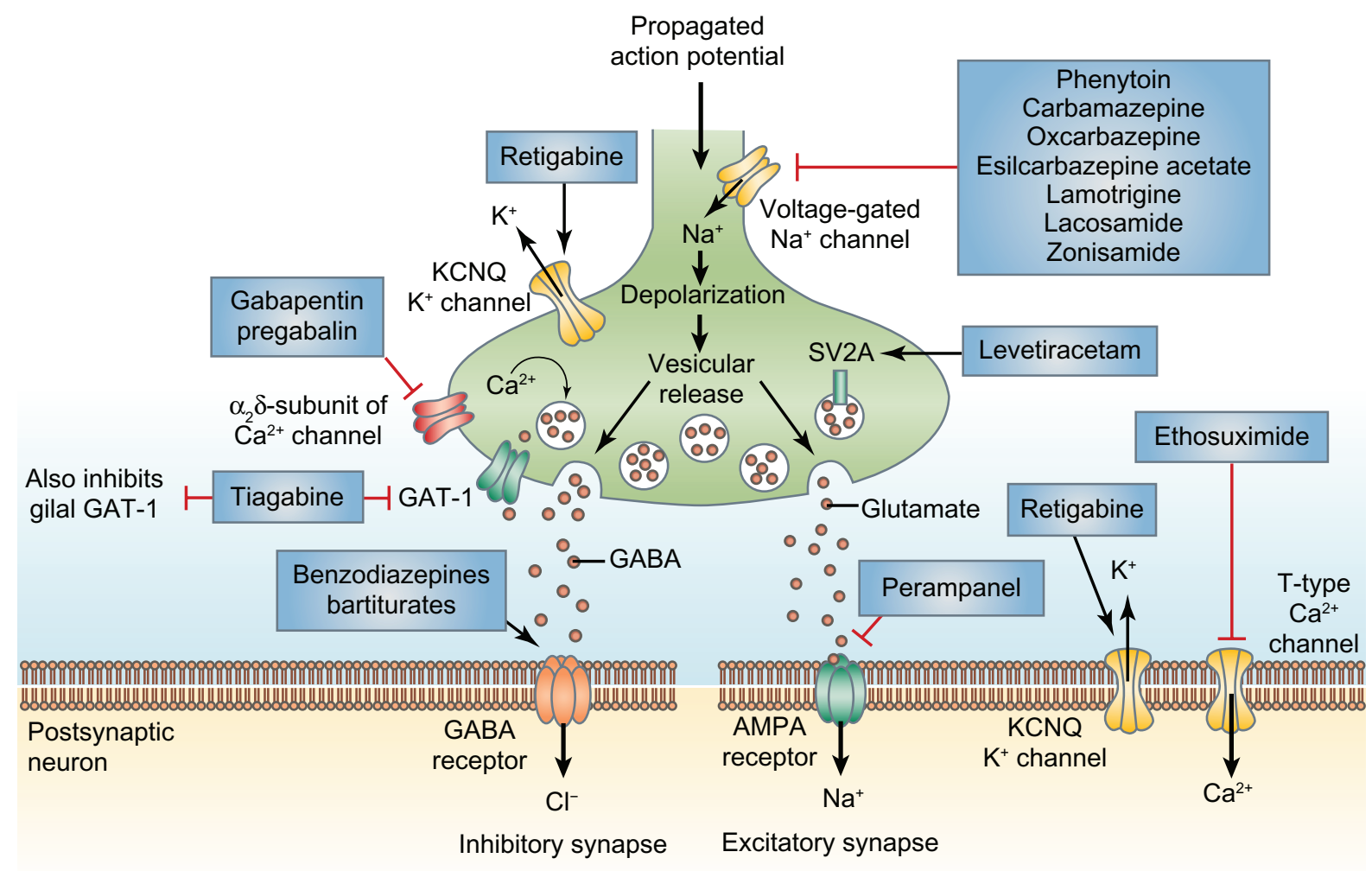

Not illustrated:

- Vigabatrin $\rightarrow \downarrow$ GABA degradation and drugs with multiple mechanisms:

- Valproate $\rightarrow \uparrow$ GABA turnover, $\downarrow \mathrm{Na}^{+}$channels, $\downarrow$ NMDA receptors

- Topiramate $\rightarrow \downarrow \mathrm{Na}^{+}$channels, $\downarrow$ AMPA/kainate receptors, $\uparrow \mathrm{GABA}_{\mathrm{A}}$ receptors

- Felbamate $\rightarrow \downarrow \mathrm{Na}^{+}$channels, $\uparrow \mathrm{GABA}_{\mathrm{A}}$ receptors, $\downarrow$ NMDA receptors

Figure I Mechanisms of action of antiepileptic drugs. Clinically approved antiepileptic drugs such as perampanel display a spectrum of mechanisms of action, with effects on both inhibitory (left-hand side) and excitatory (right-hand side) nerve terminals.

Reprinted with permission from Macmillan Publishers Ltd, Bialer M, White HS. Nat Rev Drug Discov. 2010;9:68-82.9

Abbreviations: AMPA, $\alpha$-amino-3-hydroxy-5-methyl-4-isoxazole-propionic acid; GABA, $\gamma$-aminobutyric acid; GAT-I, sodium- and chloride-depended GABA transporter I; SV2A, synaptic vesicle glycoprotein $2 \mathrm{~A}$. 
testing with Phase I studies; Phase II studies in patients with multiple sclerosis, Parkinson disease, neuropathic pain, and epilepsy; and a large Phase III program in patients with focal onset seizures. Overall, more than 4600 patients have been exposed to perampanel in clinical trials (Eisai Co, Woodcliff Lake, NJ, USA, Ltd data on file 2012).

Eisai submitted the initial marketing authorization applications to the US and European Union (EU) pharmaceutical drug regulatory agencies in January 2011. In July 2012, perampanel was granted market authorization by the European Commission as an adjunctive treatment for partial-onset seizures with or without secondarily generalized seizures in patients with epilepsy who are aged 12 years and older. ${ }^{9}$ The US Food and Drug Administration (FDA) granted approval for perampanel in October 2012 for indications identical to that granted by the EU. Its release into the general US market is expected in June-September 2013, pending formal US Drug Enforcement Agency classification as a scheduled drug.

\section{Pharmacology/mode of action}

Glutamate is the major excitatory neurotransmitter in the brain. Its receptors are postulated to play an important role in epilepsy and in many neurological conditions where excitotoxicity produces neuronal excitation and injury. Excitatory neurotransmitters including AMPA also play an important role in the pathophysiology of epilepsy, and AMPA receptors therefore represent a validated target for antiepileptic drug development. Perampanel inhibited AMPA-induced increases in intracellular $\mathrm{Ca}^{2+}$ and selectively blocks AMPA receptor-mediated synaptic transmission, thus reducing neuronal excitation. Phase I and II clinical studies revealed a favorable safety and tolerability profile that furthered the proof of concept for the safety, efficacy, and tolerability of perampanel that was observed in three Phase III clinical trials in Europe, North America, and Australia. ${ }^{10-12}$ The FDA has recommended that perampanel be classified as a scheduled drug with a potential for abuse or addiction. A boxed warning to reflect the potential for serious, possibly life-threatening, neuropsychiatric side effects is present in the package insert. Side effects include irritability, aggression, anger, anxiety, paranoia, euphoric mood, and agitation. Serious or life-threatening psychiatric and behavioral adverse reactions, including aggression, hostility, irritability, anger, and homicidal ideation and threats have been reported in patients taking perampanel. A few patients exhibited violent thoughts and threatening behavior.
Perampanel (2-[2-oxo-1-phenyl-5-pyridin-2-yl-1,2-dihydropyridin-3-yl] benzonitrile hydrate) demonstrates a broad spectrum of activity in rodent and other preclinical seizure models, unlike many of the first generation AEDs. ${ }^{13}$ The precise mechanism of action has not been fully determined. Perampanel reduces calcium influx mediated by AMPA receptors in cultured cortical neurons. In vitro studies revealed its mechanism of action as a selective noncompetitive AMPA receptor antagonist that impacts neurotransmission by high potency reduction of neuronal excitability in the brain. ${ }^{8}$ Perampanel also acts at the N-methyl-D-aspartate and the kainate receptors in the excitatory postsynaptic membranes of the neurons, though the principal ionotropic glutaminergic receptor activity involves AMPA. ${ }^{13,14}$ The in vitro concentrations of perampanel necessary to reduce AMPA receptor-mediated synaptic responses are similar to those used in clinically effective AED doses in vivo. ${ }^{14}$ In mouse models perampanel showed a protective effect against audiogenic-, pentylenetetrazol-, and maximal electroshock-induced seizures studied in monotherapy and in combination with other AEDs. ${ }^{13}$ In amygdala-kindled rats, perampanel significantly increased after discharge threshold and significantly reduced motor seizure duration, after discharge duration, and seizure severity recorded at $50 \%$ higher intensity than after discharge threshold current. Based upon its mechanism of action, perampanel has been studied in other degenerative neurological disorders where excessive glutamatergic activity has been implicated in the primary disease process. ${ }^{15}$

\section{Pharmacokinetics}

Perampanel is an orally active novel AED with a long halflife of approximately 105 hours (range, 52-129 hours in the single-dose study and 66-90 hours in the multiple-dose study) (package insert). The pharmacokinetics of perampanel is similar in healthy controls and patients with focal seizures. Perampanel is completely absorbed from the gastrointestinal tract following oral administration and rapidly reaches its maximal plasma concentration about 1 hour after ingestion. There is a negligible first-pass metabolism, and it is slowly eliminated with a steady-state plasma concentration apparent after 2 weeks. Perampanel is $95 \%$ bound to plasma proteins and is extensively metabolized via oxidation and sequential glucuronidation. It is primarily metabolized by CYP3A4 of the P450 enzyme system, though other CYP enzymes may also be involved. It does not function as an enzyme inducer or inhibitor. Enzyme-inducing AEDs including carbamazepine, oxcarbazepine, and phenytoin increased the clearance of 
perampanel and decreased its plasma concentration by at least half. Topiramate reduced the AUC of perampanel by $20 \%$, while lamotrigine, levetiracetam, valproate, zonisamide, and benzodiazepines had no effect on perampanel clearance. Perampanel reduced the clearance of oxcarbazepine by $26 \%$ and reduced the clearance of carbamazepine, valproate, and lamotrigine by $<10 \%$, though the impact on efficacy is expected to be clinically insignificant. Insufficient information exists to recommend dose adjustments to correct for this reduction. Concomitant use of perampanel with other potent CYP3A inducers (ie, rifampin and St John's wort) should be avoided to minimize drug-drug interactions. At doses of $12 \mathrm{mg} /$ day, perampanel reduced levonorgestrel by approximately 40\%; therefore, hormonal contraceptives containing levonorgestrel may be compromised with perampanel at this dose.

Perampanel is metabolized by first-order elimination pharmacokinetics. Elimination occurs primarily through the feces for approximately $70 \%$ of the dose. The remainder is excreted in the urine, with $<2 \%$ of the dose eliminated unchanged. Clearance decreased by $27 \%$ and AUC increased by $37 \%$ in mild to moderate renal dysfunction. No adjustments in mild dysfunction ( $\mathrm{CrCl} 50-80 \mathrm{~mL} / \mathrm{min}$ ) are required; however, it has not been adequately studied and is not recommended for patients with severe renal dysfunction or in patients requiring hemodialysis (HD). In hepatic disease, the AUC increased 1.8-fold in mild dysfunction and 3.3-fold in moderate dysfunction, and the drug half-life is prolonged. No effect of age on perampanel clearance was found in patients 12-74 years of age. Patients with mild hepatic impairment should have a maximum of $6 \mathrm{mg}$ daily, while those with moderate hepatic impairment should have a maximum recommended daily dose of $4 \mathrm{mg}$ once daily at bedtime. Perampanel has not yet been evaluated in patients with severe hepatic impairment. Maximum frequency for dosage increases is every 2 weeks starting at $2 \mathrm{mg} /$ day. There are no adequate and well-controlled studies in pregnant women, and perampanel is rated as Category C. Oral administration of perampanel to pregnant rats at any dose throughout organogenesis resulted in an increase in visceral abnormalities. In a dose-ranging study, embryo lethality and reduced fetal body weight were observed at the mid and high doses tested. It is unknown if perampanel is excreted in breast milk. No clear effect on fertility is known, and no evidence of mutagenesis or carcinogenesis has been seen with perampanel.

Perampanel is commercially available in round, biconvex, film-coated tablets. Tablet strengths include $2 \mathrm{mg}, 4 \mathrm{mg}, 6 \mathrm{mg}, 8 \mathrm{mg}, 10 \mathrm{mg}$, and $12 \mathrm{mg}$ formulations.
Perampanel is a white to yellowish white powder with the molecular formula $\mathrm{C}_{23} \mathrm{H}_{15} \mathrm{~N}_{3} \mathrm{O} \cdot 3 / 4 \mathrm{H}_{2} \mathrm{O}$ and molecular weight of 362.90. Population pharmacokinetics showed no age effect with exposure. It is administered in a single dose that is typically taken at night. Food slows the rate of absorption but does not affect the extent of absorption. No significant effect of sex, race, or age in patients between 12-74 years was seen. Patients older than 12 years of age may be dosed as adults. In dose-ranging studies, 2-12 $\mathrm{mg}$ of active drug per day were evaluated. A minimal effective dose of $4 \mathrm{mg}$ and a plateau in efficacy was observed at $8 \mathrm{mg}$ daily during Phase III clinical trials. ${ }^{10-12}$ No specific contraindications have been determined at this time. Information is unavailable for children younger than 12 years of age and for the geriatric population. High doses of perampanel produced euphoria that was similar to ketamine $100 \mathrm{mg}$ and alprazolam $3 \mathrm{mg}$. Official schedule designation is currently pending. A withdrawal effect has not been identified. An intentional overdose of $264 \mathrm{mg}$ has been reported. This patient experienced altered mental status, agitation, and aggressive behavior but recovered without consequence. Because of its unique mechanism of action, perampanel may be of added value in cases of refractory status epilepticus. However, there are no published reports on its use in human status epilepticus, and the lack of an intravenous formulation precludes quick administration in the initial treatment phase.

\section{Efficacy}

Two Phase II randomized, double-blind, placebo-controlled dose-escalation studies (studies 206 and 208) ${ }^{16}$ of 201 patients used pharmacokinetic and pharmacodynamics analyses to predict the range of effective doses for the Phase III studies. The minimum effective dose was $4 \mathrm{mg} /$ day, mid effective dose was $8 \mathrm{mg} /$ day, and high effective dose was $12 \mathrm{mg} /$ day. Although the studies did provide data on preliminary efficacy, they were not powered to provide conclusive data on efficacy. ${ }^{16}$ An open-label extension study ${ }^{17}$ followed up on 138 patients in studies 206 and 208 for up to 4 years. ${ }^{16}$ The average duration of exposure to perampanel was 2.2 years at doses of $2 \mathrm{mg}-12 \mathrm{mg} /$ day. The median (range) percent change in seizure frequency per 28 days relative to baseline was $-31.5 \%$ ( $-99.2 \%$ to $512.2 \%)$. The responder rate, defined as the proportion of patients experiencing a $\geq 50 \%$ reduction in seizure frequency, for all 138 patients was $37.0 \%{ }^{17}$

Three Phase III multicenter, randomized, double-blind, placebo-controlled trials (studies 304, 305, and 306 ${ }^{10-12}$ ) evaluated the efficacy of adjunctive perampanel for refractory partial-onset seizures in patients age $\geq 12$ years on one to three 
concomitant AEDs. All three trials were of similar design. Study $306^{10}$ was the first study of the three, which consisted of 706 patients and defined the minimally effective dose. The $2 \mathrm{mg} /$ day dose was not statistically different than placebo. The median percent change in seizure frequency was $-23.3 \%$ $(P=0.0026)$ and $-30.8 \%(P<0.0001)$ and responder rates were $28.5 \%(P=0.0132)$ and $34.9 \%(P=0.0003)$ for doses of $4 \mathrm{mg} /$ day and $8 \mathrm{mg}$ /day, respectively. ${ }^{10}$ Studies 304 and 305 used higher doses of perampanel. Study 304 enrolled 388 patients from North, Central, and South America and used once-daily dosing of perampanel at $8 \mathrm{mg}$ and $12 \mathrm{mg} /$ day. ${ }^{11}$ Median percent change in seizure frequency was $-26.3 \%$ $(P=0.0261)$ and $-34.5 \%(P=0.0158)$ for doses of $8 \mathrm{mg}$ and $12 \mathrm{mg} /$ day, respectively. However, the responder rates for $8 \mathrm{mg}$ and $12 \mathrm{mg} /$ day were $37.6 \%(P=0.0760)$ and $36.1 \%$ $(P=0.0914)$, respectively, and not statistically different than placebo. This was due to the fact that the placebo responder rate was much higher than expected in Central, and South American patients as compared to North American patients. The reason for this response is unexplained, but is thought to involve patient selection or study conduct. ${ }^{11}$ Study $305^{12}$ consisted of 386 patients from Europe, North America, and Australia. The median percent change in seizure frequency from baseline per 28 days was $-30.5 \%(P<0.001)$ and $-17.6 \%(P=0.011)$ and responder rates were $33.3 \%$ $(P=0.002)$ and $33.9 \%(P<0.001)$ for doses of $8 \mathrm{mg} /$ day and $12 \mathrm{mg} /$ day, respectively (Figure 2). ${ }^{18}$ During the maintenance period, $2.8 \%$ of patients in the $8 \mathrm{mg}$ group and $6.5 \%$ of the patients in the $12 \mathrm{mg}$ group became seizure free, compared to $1.7 \%$ in the placebo group. ${ }^{12}$ Therefore, results from these last two studies ${ }^{11,12}$ indicate that there was not a greater benefit for the $12 \mathrm{mg} /$ day dose versus the $8 \mathrm{mg} /$ day dose, but that doses up to $12 \mathrm{mg} /$ day may provide additional benefit in efficacy for some patients, although the numbers were too small to provide any definite conclusions. The potential efficacy difference between the $8 \mathrm{mg}$ /day dose and the $12 \mathrm{mg} /$ day dose is currently being analyzed using longterm open-label data.

Finally, an extension study of all three Phase III trials, which included 1218 patients, demonstrated that the reduction in seizure frequency in patients on adjunctive once-daily perampanel average dose of $10 \mathrm{mg}$ /day was maintained during the 1 - to 2 -year monitoring period. ${ }^{19}$ The overall median percent change in seizure frequency was $-46.5 \%$ (weeks 40-52, $\mathrm{n}=731$ ) and $-58.1 \%$ (weeks 92-104, $\mathrm{n}=59$ ), and responder rates were $46.9 \%$ and $62.7 \%$, respectively. The rate of seizure freedom was $7.1 \%$ in patients with 12 months of data. ${ }^{19}$ Some possible problems with the study are (1) loss of initial efficacy or tolerance was not examined in same-patient follow up; (2) there was no placebo comparison; and (3) changes in concomitant AEDs allowed per study protocol may have had an effect on seizure control. ${ }^{9}$

There is a positive relationship between plasma perampanel concentration and clinical response. As average exposure to perampanel at steady state increased, seizure frequency decreased and the probability of an individual being a responder ( $\geq 50 \%$ reduction in seizure frequency) increased significantly. Concomitant AEDs had no effect on the exposure/efficacy relationship for seizure frequency or the probability of response at any given plasma concentration. ${ }^{20}$

\section{Safety/tolerability}

Treatment with adjunctive perampanel is safe, and tolerability is acceptable. Doses of 4-12 mg/day were well tolerated both once and twice daily in two Phase II dose-escalation studies, and the most common side effects reported in study $208^{16}$ were dizziness (57.9\%), somnolence (31.6\%), and headache (18.4\%). Fatigue, diarrhea, and rhinitis were all reported in $10.5 \%$ of patients. The adverse effects were of mild to moderate severity. No serious adverse effects or deaths were reported. ${ }^{16}$ In the open-label extension study, no new safety concerns were identified; the most common treatmentemergent adverse events (TEAEs) occurring over 4 years were dizziness $(41.3 \%)$, headache $(21 \%)$, and somnolence (19.6\%). These common TEAEs decreased significantly over a 4 -year period (ie, dizziness, $30.4 \%$ at year 1 versus $6.7 \%$ at year 4; somnolence, $17.4 \%$ at year 1 versus $1.7 \%$ at year 4 ; headache, $16.7 \%$ at year 1 versus $5.0 \%$ at year 4$).{ }^{17}$ Mild anxiety was the only psychiatry-related TEAE, occurring in $7.2 \%$ of patients. No TEAE related to suicidality was reported. Overall, $12.3 \%$ of patients withdrew from the study due to TEAEs. ${ }^{17}$

Of the three Phase III studies, the most common TEAEs with the $8 \mathrm{mg} /$ day dose were dizziness $(26.6 \%-37.6 \%)$, somnolence $(12.4 \%-18.0 \%)$, headache $(8.5 \%-15.0 \%)$, and fatigue $(5.3 \%-13.2 \%),{ }^{10-12}$ with a dose-related effect observed for all with the exception of headache. Less frequent TEAEs at the $8 \mathrm{mg}$ /day dose included falls (9.8\%), irritability $(7.5 \%)$, and ataxia $(6.0 \%)$. Weight increases of $>7 \%$ $(1.6 \mathrm{~kg}-1.9 \mathrm{~kg})$ were noted in $19.2 \%$ of perampanel patients at doses of $8 \mathrm{mg}$ and $12 \mathrm{mg} /$ day in one study ${ }^{11}$ but $5.6 \%$ and $6.8 \%$ in other studies. ${ }^{12,19}$ Depression, psychosis, and rash were not statistically different than placebo. The rates of rash were low and ranged from $1.7 \%-3.2 \%$, and there were no reports of Stevens-Johnson syndrome. Discontinuations due to TEAEs were $8.5 \%$ and $19.0 \%$ at doses of $8 \mathrm{mg} /$ day 
A

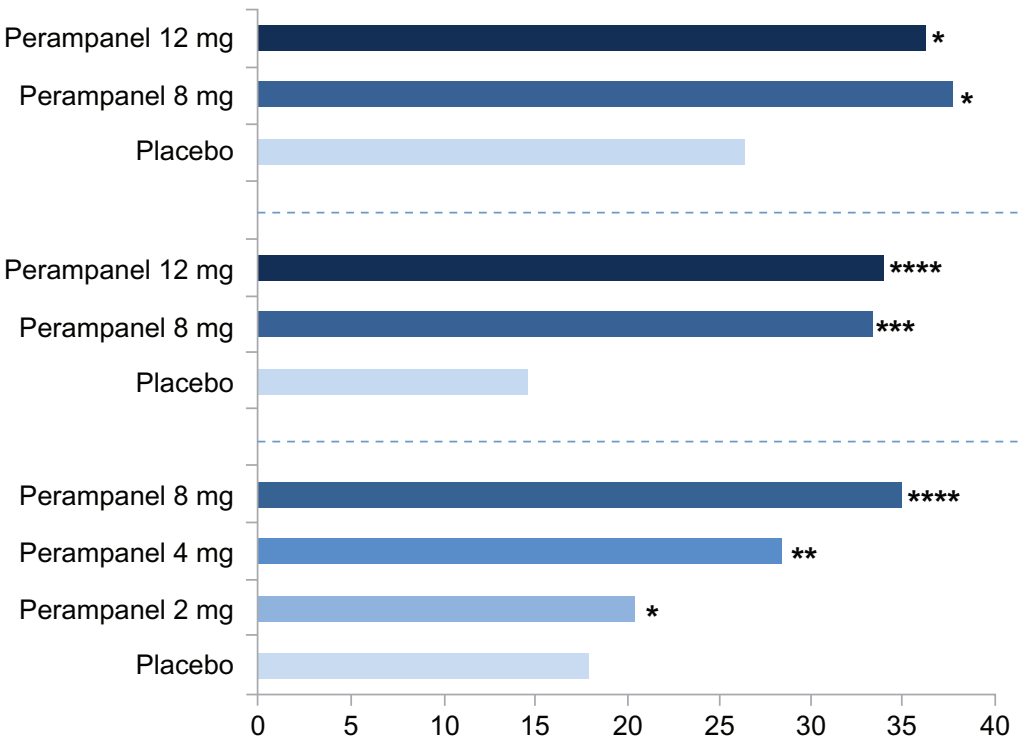

B

Perampanel $12 \mathrm{mg}$

Perampanel $8 \mathrm{mg}$

Placebo

Perampanel $12 \mathrm{mg}$

Perampanel $8 \mathrm{mg}$

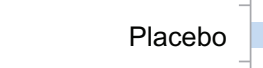

Perampanel $8 \mathrm{mg}$

Perampanel $4 \mathrm{mg}$

Perampanel 2 mg

Placebo

\section{Median \% change in seizure frequency}
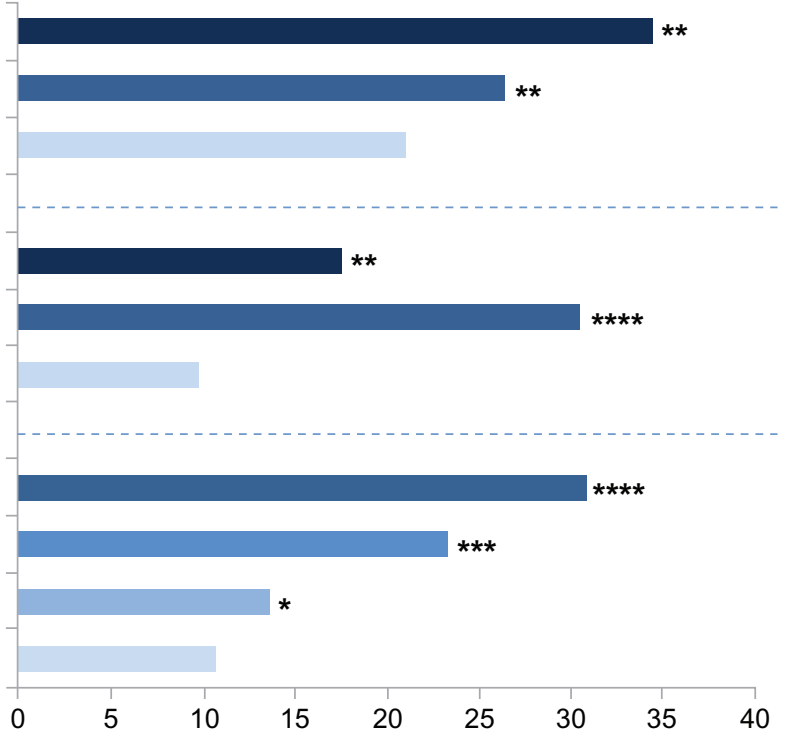

**
Study 304

Study 305

Study 306

Study 304

Study 305

Study 306

Figure 2 (A) 50\% responder rate (percentage of patients achieving a 50\% or greater reduction in seizure frequency) during treatment with perampanel as adjunctive therapy; (B) median percentage change in seizure frequency per 28 days during treatment relative to baseline over the 19-week double-blind period in studies 304 ( $n=388$ ), ${ }^{6}$ $305(\mathrm{n}=386)^{7}$ and $306(\mathrm{n}=706) .{ }^{8} * \mathrm{P}=$ non significant; $* * p<0.05 ; * * * P<0.005 ; * * * * P<0.00$ I versus placebo.

Reprinted with permission from Academic Press. Franco V, Crema F, ludice A, Zaccara G, Grillo E. Novel treatment options for epilepsy: focus on perampanel. Pharmacol Res. 2013;70(1):35-40. ${ }^{18}$

and $12 \mathrm{mg} /$ day, respectively, which is consistent with the discontinuation rates of other recently approved AEDs, lacosamide and retigabine, at the highest doses. ${ }^{12}$ Retention rates were $>70 \%$ after approximately 1 year of treatment, and concomitant intake of multiple AEDs did not affect the tolerability of perampanel. ${ }^{19}$ No clinically important changes in the clinical exams, vital signs, laboratory values, or electrocardiogram (EKG)s were observed. Abuse or diversion of perampanel was not reported. Human studies regarding fertility and teratogenesis are not yet available. In a recent meta-analysis comparing some of the newer AEDs (including eslicarbazepine, retigabine, carisbamate, lacosamide, brivaracetam, and perampanel), the odds ratio for withdrawal rate of perampanel was 0.50 , which indicates better tolerability of perampanel compared to all the other newer AEDs except brivaracetam. ${ }^{21}$ The different clinical trials design for the 
different AEDs preclude a head-to-head comparison, and the meta-analysis approach has limitations. Nevertheless, perampanel is likely to fall into the group of AEDs that is well-tolerated by the vast majority of patients.

\section{Patient-focused perspectives}

Perampanel is a new type of AED with an encouraging clinical profile based upon a design that inhibits excitatory amino acids that are linked to epileptic seizure generation and spread. ${ }^{22}$ Unique theoretical concerns for perampanel include behavioral and psychiatric side effects because of the drug's mechanism of action (similar to phencyclidine, or PCP). The use of perampanel should be monitored to assess patients for temporal signs of anger; aggression; unfavorable changes in mood, personality or behavior; and other behavioral symptoms, including the emergence of suicidal thoughts or gestures. Monitoring patients for psychiatric and behavioral reactions should be performed, especially during titration and at higher doses. These symptoms should be reported immediately to their physician or health care provider. However, depression, psychosis, and suicidality were limited or even absent in some clinical trials, with a psychiatric profile overall that was comparable to placebo. ${ }^{12}$ Perampanel should be reduced if this occurs; if symptoms are severe or worsening, then it should be discontinued immediately. Withdrawing the drug seems to produce no unexpected withdrawal-like symptoms, though abrupt discontinuation may increase seizure frequency. Patients should be advised not to drive or operate machinery until sufficient experience on perampanel has been attained, especially if is coadministered with other central nervous system (CNS) depressant medications, including alcohol. In the absence of coadministered enzyme-inducing antiepileptic drugs, the initial starting dose is $2 \mathrm{mg}$ orally at bedtime (4 mg with enzyme-inducing antiepileptic drugs). This may be increased by $2 \mathrm{mg} /$ day every week to a total of 4 to $8 \mathrm{mg}$ /day. The maximum recommended daily dose is $12 \mathrm{mg}$ at bedtime. Somnolence and dizziness are most frequently encountered side effects, though other side effects have been notably inconspicuous. Weight gain has been observed during treatment with perampanel. Like the effect seen in many other AEDs, higher doses of perampanel may result in a greater likelihood of side effects and necessitate dosage adjustment. Still, most patients reported side effects as mild or moderate. In healthy volunteers, the effects of perampanel did not impair simple psychomotor tasks, driving performance, or motor coordination. Other rare serious side effects seen with AEDs, including liver or bone marrow failure and life-threatening rash, have thus far not been identified with perampanel. Falls were encountered more frequently in patients. Some occurred in those with higher serum concentrations of the drug, with other CNS side effects such as dizziness, the same day as convulsions, and often recurred in the same day. ${ }^{12}$ This association, while meriting further study, should be taken into consideration in patients with gait dysfunction or perhaps in the elderly at high risk for fall-related injury. Less than $10 \%$ note seizure exacerbation with effective dosing, and serious adverse events when encountered are usually related to epilepsy. ${ }^{19}$ Laboratory testing, changes in vital signs, and altered cardiac function noted on EKG parameters have not been observed; therefore, seizure control is indicative of efficacy and routine serologic monitoring is not required. As noted above, over $70 \%$ of patients in an open label study remained on perampanel at the 1-year mark. Even after 3-4 years of perampanel therapy, over one-third of patients still continued to take the medication. This favorable retention rate reflects patient satisfaction as a result of treatment. ${ }^{17} \mathrm{~A}$ singledaily dose regimen with perampanel enhances the likelihood that patients will adhere to AED therapy. ${ }^{23}$ In elderly patients and in patients sensitive to medication, dose escalation every 2 weeks may help improve tolerability. About one-third of patients suffer from drug-resistant seizures, and even more have a lower quality of life due to AED side effects. ${ }^{24}$ When enzyme-inducing AEDs are introduced or withdrawn from a patient taking perampanel, the patient should be counseled on, and monitored for, any adverse effects which may necessitate perampanel dose adjustment. Because of the unique pharmacology, favorable pharmacokinetics, and clinical profile, perampanel should be a useful option in adolescent and adult patients with epilepsy.

\section{Potential antiepileptogenic effect}

Prevention of epileptogenesis remains a significant unmet medical need in the field of epilepsy. ${ }^{25}$ Most of the marketed AEDs have been developed primarily in models of acute seizures (not of epileptogenesis) and do not have antiepileptogenic effects. Several groups hypothesize that drugs with antiepileptogenic properties may significantly reduce the numbers of patients developing medically refractory epilepsy. ${ }^{26-28}$ A potentially promising therapeutic target to prevent epileptogenesis is the AMPA-type glutamate receptor (AMPAR). In a rat model of neonatal seizures, intraperitoneal administration of AMPAR antagonists within 48 hours of early-life seizures reduced later-life seizure susceptibility and hippocampal neural injury. ${ }^{29}$ Administration of AMPAR antagonists after seizures reduced kinase activity and phosphorylation of AMPAR subunits, thereby preventing an 
increase in seizure susceptibility. ${ }^{30}$ Therefore, changes in AMPAR phosphorylation seem important for epileptogenesis and antagonism of these changes may have antiepileptogenic effects. $^{31}$

Autophagy is important in the regulation of inflammation and the coordination of innate and adaptive immune responses. ${ }^{32}$ The possible involvement of impaired autophagy and inflammation in epileptogenesis raises the question of whether these processes play a collaborating role in the development of seizures. Neuronal stimulation induces autophagy in hippocampal neurons that is involved in AMPA receptor degradation. ${ }^{33}$ Impaired autophagy contributes to epileptogenesis in a mouse model. ${ }^{34}$ The connections between autophagy and inflammation are complex, and much remains to be worked out. However, AMPAR subunit expression appears related to autophagy and inflammatory processes, ${ }^{31}$ and modulation of this receptor complex may be antiepileptogenic. Whether an AMPAR antagonist can help prevent the development of epilepsy remains to be seen. But the evidence from animal data holds out hope that perampanel has both antiseizure and antiepileptogenic effects.

\section{Conclusion}

Perampanel is the first AMPA-receptor antagonist marketed for the treatment of partial onset seizures with or without secondary generalization. Its efficacy in clinical trials is similar to that of AEDs approved for use in the past decade. The proportion of patients rendered seizure-free in the clinical trials was about 7\%, which is also in line with data from drug trials of other AEDs. Thus, perampanel does not appear to confer significantly better seizure control than other AEDs available in the US and EU. Perampanel is considered a safe drug with an acceptable tolerability profile. The most common side effects are well-known to physicians using AEDs, and include the usual suspects such as dizziness, somnolence, headache, and fatigue. Anxiety and irritability may be seen in some patients taking perampanel, but there was no evidence of increased risk for suicidality, psychosis or major depression. With this efficacy and side effect profile, perampanel promises to be a useful addition to the armamentarium of the physician treating epilepsy patients. Two pharmacologic properties of perampanel suggest the possibility that the drug may become a first-tier AED. Its unique mechanism of action opens up an entirely new option for practitioners of rational polytherapy. Perampanel's potential antiepileptogenic effects also warrant further investigation into its use early on in the disease process. Ultimately, where the drug fits in the epilepsy treatment algorithm will depend largely on the responses of the first $25,000-50,000$ patients and the experiences of the physicians prescribing for them.

\section{Disclosure}

The authors report no conflicts of interest in this work.

\section{References}

1. French JA, Kanner AM, Bautista J, et al. Efficacy and tolerability of the new antiepileptic drugs II: treatment of refractory epilepsy: report of the Therapeutics and Technology Assessment Subcommittee and Quality Standards Subcommittee of the American Academy of Neurology and the American Epilepsy Society. Neurology. 2004;62(8):1261-1273.

2. Glauser T, Ben-Menachem E, Bourgeois B, et al. Updated ILAE evidence review of antiepileptic drug efficacy and effectiveness as initial monotherapy for epileptic seizures and syndromes. Epilepsia. 2013;54(3):551-563.

3. Perucca E, French J, Bialer M. Development of new antiepileptic drugs: challenges, incentives, and recent advances. Lancet Neurol. 2007;6(9): 793-804.

4. French JA, Faught E. Rational polytherapy. Epilepsia. 2009; 50(Suppl 8):63-68.

5. Bialer M, Johannessen SI, Levy RH, Perucca E, Tomson T, White HS. Progress report on new antiepileptic drugs: a summary of the Eleventh Eilat Conference (EILAT XI). Epilepsy Res. 2013;103(1):2-30.

6. Beyreuther BK, Freitag J, Heers C, Krebsfanger N, Scharfenecker U, Stohr T. Lacosamide: a review of preclinical properties. CNS Drug Rev. 2007;13(1):21-42.

7. Bialer M, Johannessen SI, Levy RH, Perucca E, Tomson T, White HS. Progress report on new antiepileptic drugs: a summary of the Tenth Eilat Conference (EILAT X). Epilepsy Res. 2010;92(2-3):89-124.

8. Rogawski MA. Revisiting AMPA receptors as an antiepileptic drug target. Epilepsy Curr. 2011;11(2):56-63.

9. Loscher W, Schmidt D. Epilepsy: perampanel-new promise for refractory epilepsy? Nat Rev Neurol. 2012;8(12):661-662.

10. Krauss GL, Serratosa JM, Villanueva V, et al. Randomized phase III study 306: adjunctive perampanel for refractory partial-onset seizures. Neurology. 2012;78(18):1408-1415.

11. French JA, Krauss GL, Biton V, et al. Adjunctive perampanel for refractory partial-onset seizures: randomized phase III study 304. Neurology. 2012;79(6):589-596.

12. French JA, Krauss GL, Steinhoff BJ, et al. Evaluation of adjunctive perampanel in patients with refractory partial-onset seizures: results of randomized global phase III study 305. Epilepsia. 2013;54(1):117-125.

13. Hanada T, Hashizume Y, Tokuhara N, et al. Perampanel: a novel, orally active, noncompetitive AMPA-receptor antagonist that reduces seizure activity in rodent models of epilepsy. Epilepsia. 2011;52(7):1331-1340.

14. Ceolin L, Bortolotto ZA, Bannister N, Collingridge GL, Lodge D, Volianskis A. A novel anti-epileptic agent, perampanel, selectively inhibits AMPA receptor-mediated synaptic transmission in the hippocampus. Neurochem Int. 2012;61(4):517-522.

15. Duty S. Targeting glutamate receptors to tackle the pathogenesis, clinical symptoms and levodopa-induced dyskinesia associated with Parkinson's disease. CNS Drugs. 2012;26(12):1017-1032.

16. Krauss GL, Bar M, Biton V, et al. Tolerability and safety of perampanel: two randomized dose-escalation studies. Acta Neurol Scand. 2012;125(1):8-15.

17. Rektor I, Krauss GL, Bar M, et al. Perampanel Study 207: long-term open-label evaluation in patients with epilepsy. Acta Neurol Scand. 2012;126(4):263-269.

18. Franco V, Crema F, Iudice A, Zaccara G, Grillo E. Novel treatment options for epilepsy: focus on perampanel. Pharmacol Res. 2013; 70(1):35-40. 
19. Krauss GL, Perucca E, Ben-Menachem E, et al. Perampanel, a selective, noncompetitive alpha-amino-3-hydroxy-5-methyl-4-isoxazolepropionic acid receptor antagonist, as adjunctive therapy for refractory partialonset seizures: interim results from phase III, extension study 307. Epilepsia. 2013;54(1):126-134.

20. Laurenza A, Ferry J, Hussein Z. Population pharmacokinetics and pharmacodynamics of perampanel: a pooled analysis from three Phase III trials. Proceedings of the American Epilepsy Society 65th Annual Meeting; December 2-6, 2011; Baltimore, MD, USA. West Hartford, CT, USA: American Epilepsy Society, 2011.

21. Gao L, Xia L, Zhao FL, Li SC. Clinical efficacy and safety of the newer antiepileptic drugs as adjunctive treatment in adults with refractory partial-onset epilepsy: a meta-analysis of randomized placebo-controlled trials. Epilepsy Res. 2013;103(1):31-44.

22. Rogawski MA, Hanada T. Preclinical pharmacology of perampanel, a selective non-competitive AMPA receptor antagonist. Acta Neurol Scand Suppl. 2013;127(Suppl 197):19-24.

23. Cramer JA, Mattson RH, Prevey ML, Scheyer RD, Ouellette VL. How often is medication taken as prescribed? A novel assessment technique. JAMA. 1989;261(22):3273-3277.

24. Kwan P, Brodie MJ. Early identification of refractory epilepsy. $N$ Engl J Med. 2000;342(5):314-319.

25. Simonato M, Loscher W, Cole AJ, et al. Finding a better drug for epilepsy: preclinical screening strategies and experimental trial design. Epilepsia. 2012;53(11):1860-1867.

26. Kobow K, Auvin S, Jensen F, et al. Finding a better drug for epilepsy: antiepileptogenesis targets. Epilepsia. 2012;53(11):1868-1876.
27. Loeb JA. Identifying targets for preventing epilepsy using systems biology. Neurosci Lett. 2011;497(3):205-212.

28. Loscher W, Schmidt D. Modern antiepileptic drug development has failed to deliver: ways out of the current dilemma. Epilepsia. 2011;52(4):657-678.

29. Sanchez RM, Dai W, Levada RE, Lippman JJ, Jensen FE. AMPA/ kainate receptor-mediated downregulation of GABAergic synaptic transmission by calcineurin after seizures in the developing rat brain. J Neurosci. 2005;25(13):3442-3451.

30. Rakhade SN, Zhou C, Aujla PK, Fishman R, Sucher NJ, Jensen FE. Early alterations of AMPA receptors mediate synaptic potentiation induced by neonatal seizures. J Neurosci. 2008;28(32):7979-7990.

31. Bakker J, Basedow FJ, Dekker AD, Papantoniou C. Phosphorylation of AMPA-type glutamate receptors: the trigger of epileptogenesis? J Neurosci. 2013;33(14):5879-5880.

32. Jo EK, Shin DM, Choi AM. Autophagy: cellular defense to excessive inflammation. Microbes Infect. 2012;14(2):119-125.

33. Shehata M, Matsumura $\mathrm{H}$, Okubo-Suzuki R, Ohkawa $\mathrm{N}$, Inokuchi K. Neuronal stimulation induces autophagy in hippocampal neurons that is involved in AMPA receptor degradation after chemical long-term depression. J Neurosci. 2012;32(30):10413-10422.

34. McMahon J, Huang X, Yang J, et al. Impaired autophagy in neurons after disinhibition of mammalian target of rapamycin and its contribution to epileptogenesis. J Neurosci. 2012;32(45):15704-15714.
Therapeutics and Clinical Risk Management

\section{Publish your work in this journal}

Therapeutics and Clinical Risk Management is an international, peerreviewed journal of clinical therapeutics and risk management, focusing on concise rapid reporting of clinical studies in all therapeutic areas, outcomes, safety, and programs for the effective, safe, and sustained use of medicines. This journal is indexed on PubMed Central, CAS,

\section{Dovepress}

EMBase, Scopus and the Elsevier Bibliographic databases. The manuscript management system is completely online and includes a very quick and fair peer-review system, which is all easy to use. Visit http://www.dovepress.com/testimonials.php to read real quotes from published authors.

Submit your manuscript here: http://www.dovepress.com/therapeutics-and-clinical-risk-management-journal 